\title{
Frontières
}

\section{Des rituels funéraires à la fête patronale Les miroloyia, lamentations vocales et instrumentales de l'Épire, Grèce}

\section{Hélène Delaporte}

Volume 20, numéro 2, printemps 2008

Les musiques et la mort

URI : https://id.erudit.org/iderudit/018335ar

DOI : https://doi.org/10.7202/018335ar

Aller au sommaire du numéro

Éditeur(s)

Université du Québec à Montréal

ISSN

1180-3479 (imprimé)

1916-0976 (numérique)

Découvrir la revue

Citer cet article

Delaporte, H. (2008). Des rituels funéraires à la fête patronale Les miroloyia, lamentations vocales et instrumentales de l'Épire, Grèce. Frontières, 20(2),

55-59. https://doi.org/10.7202/018335ar
Résumé de l'article

En Épire, le terme miroloyia désigne deux types de lamentations appartenant à des contextes très différents. Les premières sont le fait des femmes qui se lamentent lors des rituels funéraires ou au quotidien en cachette des hommes, les secondes sont jouées par des musiciens tsiganes dans les fêtes patronales. L'article s'interroge sur l'articulation entre ces deux formes de miroloyia et met en évidence le rôle différencié des femmes et des hommes face à la mort. Si les femmes gèrent la mort alors qu'elle vient de surgir et s'adressent directement aux défunts, les hommes sont alors très en retrait. En revanche, c'est à l'occasion des fêtes patronales que les hommes pleurent leurs morts. Ils ne s'adressent pas directement à eux mais passent par un intermédiaire, le clarinettiste tsigane, à qui ils confient la gestion de leurs émotions. 


\section{SURLE

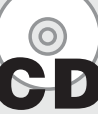

Extrait de la plage $1 \mathrm{du}$ disque Grèce, Koumpania Xalkias, Musiciens traditionnels d'Épire, collection Inédit Maison des Cultures du Monde, W260117, 2004.

Durée: $4 \min 20 \mathrm{~s}$

Résumé

En Épire, le terme miroloyia désigne deux types de lamentations appartenant à des contextes très différents. Les premières sont le fait des femmes qui se lamentent lors des rituels funéraires ou au quotidien en cachette des hommes, les secondes sont jouées par des musiciens tsiganes dans les fêtes patronales. L'article s'interroge sur l'articulation entre ces deux formes de miroloyia et met en évidence le rôle différencié des femmes et des hommes face à la mort. Si les femmes gèrent la mort alors qu'elle vient de surgir et s'adressent directement aux défunts, les hommes sont alors très en retrait. En revanche, c'est à l'occasion des fêtes patronales que les hommes pleurent leurs morts. Ils ne s'adressent pas directement à eux mais passent par un intermédiaire, le clarinettiste tsigane, à qui ils confient la gestion de leurs émotions.

Mots clés: miroloyia - lamentation fête - tsigane - mort - exil - Grèce.

\section{Abstract}

In Epirus, the word miroloyia refers to two types of laments, performed in very different circumstances. The former are women's laments, occurring during funerary rituals or, unknown from men, in everyday life; the latter are played by gypsy musicians during patronal feasts. This article questions the link between both types of miroloyia and shows up the differentiated roles of women and men as regards death. Women manage death when it has just happened and speak directly to the dead persons, while men stand aside. On the other hand, patronal feasts give men the opportunity to weep over their dead. They do not directly speak to them but do it through an intermediary, the gypsy clarinet player, whom they entrust with managing their emotions.

Keywords: miroloyia - lament - feast gypsy - death - exile - Greece.

\section{Des rituels funéraires} à la fête patronale Les miroloyia, lamentations vocales et instrumentales de l'Épire, Grèce

\author{
Hélène Delaporte, Ph. D., \\ Centre de recherche en ethnomusicologie, \\ Musée de I'Homme, CNRS UMR 7186 LESC, \\ Université Paris X Nanterre.
}

Dans l'ensemble de la Grèce, le terme mirolö̈ signifie littéralement «discours sur le destin», de mira, destin et logos, discours. Il désigne dans tout le pays les lamentations des femmes à l'occasion des rituels funéraires.

En Épire, région du nord-ouest de la Grèce, le terme est aussi employé pour désigner des lamentations instrumentales. Ces dernières sont jouées par des musiciens tsiganes lors des fêtes patronales appelées paniyiria.

Ainsi, ces deux types de lamentations, si elles portent le même nom, appartiennent à des contextes différents qui ne se recoupent pas. Comment s'articulent lamentations vocales et lamentations instrumentales? Comment comprendre la présence de miroloyia dans les fêtes patronales? Qu'est-ce que leur étude nous apprend sur la façon d'appréhender la mort en Épire ${ }^{1}$ ?

\section{LA LAMENTATION VOCALE, UN OBJET QUI SE DÉROBE}

Après plusieurs terrains successifs dans le nord de l'Épire, où j'étais perçue comme «la fille qui s'intéresse à la musique traditionnelle et qui dort chez les tsiganes», je revins en annonçant mon intérêt pour les lamentations funéraires. "Ça ne se fait plus, on est en Europe maintenant!», «Tu arrives trop tard! Plus personne ne les connaît» me disaient la plupart de mes interlocuteurs; se pouvait-il que cette pratique, encore observée dans les années quatre-vingt (Auerbach, 1987; Caravelli, 1980), ait totalement disparu?

Un deuxième type de réactions était pour le moins intrigant. "Pourquoi tu les veux ces choses pour les morts?», me reprochait le clarinettiste qui m'accueille habituellement, sur un ton effrayé. Mon intérêt pour les lamentations semblait vraiment lui poser problème. Lorsque quelqu'un l'interrogeait sur la raison de ma présence chez lui, soit il esquivait, soit il présentait les choses ainsi: "C'est l'université de Paris qui nous l'envoie, c'est 
son professeur qui l'oblige à travailler sur les lamentations. » Que ce soit une initiative personnelle n'était pas acceptable. Le plus souvent, l'interlocuteur ne relevait pas, changeait de sujet avant que de s'esquiver.

Si je n'avais encore aucune indication sur la pratique réelle des lamentations, force était de constater que le mot miroloï ne laissait personne indifférent. D'une manière ou d'une autre, il résonnait encore. En réalité plus l'objet se dérobait, plus il se faisait présent. La force du mirolö̈ se percevait dans les regards désapprobateurs, voire craintifs des villageois.

Après plusieurs semaines et à force d'exprimer mon désarroi, le même clarinettiste finit par me dire innocemment:«Et Matoula, tu l'as vue? C'est elle qui connaît tout ça.»Sa femme, qui m'avait affirmé ne connaître personne qui puisse m'aider, fit un «ah?» dubitatif. Non seulement je connaissais Matoula, mais nous étions allées plusieurs fois lui rendre visite. La crainte que provoquait mon intérêt pour les miroloyia leur avait fait dissimuler ce qu'ils savaient parfaitement.

Matoula, une femme de soixante-quatorze ans, est en effet ce qu'on appelle une miroloyistria, une pleureuse. Avec elle et quelques autres miroloyistres, je pus enfin m'entretenir de la pratique des lamentations. Les pleureuses ne sont pas professionnelles, dans le sens où elles ne sont pas rémunérées, mais sont des spécialistes: venues en voisines, elles soutiennent les parentes du défunt dans leurs lamentations et veillent au bon déroulement du rituel. Toute femme n'est pas forcément une bonne pleureuse et le fait d'être avancée en âge ne constitue pas le critère premier. Il faut avant tout, me dit l'une d'entre elles, "avoir connu beaucoup de malheur dans sa vie».

\section{UNE PRATIQUE RITUELLE ET PROFANE}

En Épire comme dans le reste de la Grèce, la mort est donc une affaire de femmes. Ce sont elles qui pleurent le défunt durant la veillée mortuaire, qui l'accompagnent de leurs lamentations jusqu'au cimetière et lors de la mise en terre. Â côté de la gestion immédiate de la mort survenue, les femmes jouent encore un rôle de premier plan lors d'autres rituels liés au devenir de l'âme du défunt.

Des cérémonies de commémoration, mnimosina, sont réparties dans le temps suivant la conception orthodoxe du chemin accompli par Jésus après sa mort. Les plus importantes sont appelées stis tris et stis saranda, littéralement "à trois» et «à quarante». Ainsi, trois jours après les funérailles, une messe est célébrée pour le défunt qui est à nouveau pleuré par les femmes autour de la tombe. Cela correspond à l'intervalle entre la mise au tombeau du Christ et sa résurrection. Avant ce délai, personne n'a le droit de se rendre au cimetière. La célébration stis saranda, obéissant au même schéma rituel, a lieu quarante jours après le décès, période durant laquelle le Christ apparaît à ses disciples avant de monter au Ciel. Six mois puis un an après le décès se tiennent d'autres commémorations semblables mais qui semblent avoir moins d'importance (Danforth, 1982, p. 44-45).

\section{HORS RITUEL, \\ PLEURER SES MORTS, \\ C'EST RISQUER \\ D'ATTIRER LA MORT.}

Enfin, les orthodoxes pratiquent l'exhumation du corps du défunt, i ektafi, littéralement «hors de la tombe» (voir Alexiou, 1974, p. 48). Cela se fait dans un délai variant en général entre trois et cinq ans après le décès. Ce rituel est central car c'est seulement avec l'exhumation que les villageois sont assurés que l'âme du défunt est bien partie. Loring Danforth écrit: "As a person's flesh (the impure, perishable portion of the body) decomposes, his sins are forgiven. At the end of this parallel processes both the body and soul exist in a pure and permanent form » (1982, p. 49). Le mort est déterré et ses os examinés. S'ils sont bien blancs, que ses cheveux se sont décomposés alors son âme n'errera pas sur terre. Si les os «ne sont pas bien sortis»(dhen vikan kala), c'est que le défunt «n'avait pas une bonne âme» (dhen ikhé kali psikhi) et risque de devenir un vrikolakas, un vampire ou un revenant. Durant toutes les étapes de l'exhumation, les proches parentes mais aussi d'autres femmes font entendre leurs lamentations. Le pope clôt ensuite le rituel par une prière ${ }^{2}$.

Les lamentations des femmes se pratiquent en parallèle des rituels orthodoxes. Tout comme l'a très bien montré Anna Serementakis dans le Magne, l'on ne confond pas les rituels de deuil et le rituel religieux (Serementakis, 1991, p. 161). L'antagonisme latent entre ces rites (Alexiou, 1974) peut parfois s'exprimer violemment. Ce fut le cas lors d'une cérémonie de quarante jours à laquelle j'ai pu assister. Pendant que le pope officiait dans l'église, une petite dizaine de femmes se trouvait au cimetière attenant. Certaines bavardaient, d'autres sanglotaient discrètement, et quatre d'entre elles se lamentaient. Le mari de l'une d'elles fit irruption en criant : «Arrêtez-vous! C'est le pope qui m'envoie! Vous avez un mnimossino maintenant! Ça suffit! [ ] Non mais qu'est-ce que c'est que ça!» Le pope les rappelait à l'ordre: le véritable rituel se passe à l'église. Elles acquiescèrent sans pour autant obtempérer sur-le-champ. L'une d'elles m'expliqua plus tard: "Sans miroloyia, ça ne se fait pas! [] Tu ne peux pas laisser le mort comme ça. Il a besoin de miroloyia, tu dois les lui dire.» Elle évita toutefois de commenter l'attitude du pope.

En dehors des rituels, certaines femmes ont une pratique des miroloyia plus personnelle. Seules, une fois par semaine ou davantage, elles se rendent au cimetière pour entretenir la tombe de leurs morts et, si elles le souhaitent, elles se lamentent. Ainsi que me l'a dit l'une d'entre elles, «tu pleures sans même le vouloir [ ] quand tu souffres les mots te viennent tout seuls ».

Mais il existe une pratique plus intime encore: certaines se lamentent chez elles quand elles en ressentent le besoin et surtout, comme nous le verrons, quand elles le peuvent. Les lamentations obéissent à une temporalité dans les rituels, de même au quotidien elles ne peuvent surgir n'importe quand.

\section{ENTRE CRAINTE ET FASCINATION}

Hors rituel, pleurer ses morts, c'est risquer d'attirer la mort. Alors qu'une vieille dame était supposée m'avoir dit des miroloyia, sa belle-fille enceinte de huit mois se plaignit publiquement de douleurs et s'interrogea à voix haute: "Tu crois que c'est à cause d'elle et ses histoires? », me désignant.

Cet ami clarinettiste, qui avait fini par me conduire chez une vieille dame afin que je puisse l'enregistrer, se plaignit le lendemain d'une vive douleur au cœur alors que nous plantions des oignons. "Tu crois que c'est parce que je l'ai accompagnée hier? » demanda-t-il à sa femme sans vraiment attendre de réponse. Celle-ci trouva l'idée plutôt amusante.

Autre anecdote, plus radicale encore. Le lendemain du jour où j'ai enregistré Matoula pour la première fois, le fils de la maison me dit:

Tu sais ce qui est arrivé à Matoula?

- Non.

- Elle est morte!

Alors que je restais interdite, toute la famille partit d'un grand rire général. Derrière cette plaisanterie, le message était bien passé : je serai bien inspirée de ne pas m'intéresser de trop près à tout cela; mes enquêtes finiraient pas faire du tort.

Cette superstition, et la réprobation qui entoure cette pratique, est surtout le 
fait des hommes. Hors rituel, les femmes se lamentent en cachette de ces derniers (voir aussi Caravelli, 1980). Ainsi, pour enregistrer Matoula, il fallait que je vienne à un moment où son frère serait absent de la maison paternelle où, tous deux célibataires, ils vivent ensemble. La femme du musicien chez qui j'habitais m'avertit: «Allez! Vas-y, c'est le moment! Son frère est parti! Fais bien attention, tu sais qu'il peut piquer des colères! »

Alors que je l'enregistrais, son frère rentra plus tôt que prévu. Immédiatement elle se justifia: "Ah! Vassilis, on est en train de dire des vieilles chansons avec Hélène. » Elle tentait ainsi de prévenir sa colère car, en Épire, comme dans toute la Grèce, chanson et lamentation s'opposent (Herzfeld, 1981). "Alo to traghoudhi, alo to mirolö̈» (la chanson est une chose, le miroloi en est une autre), selon l'expression de Matoula. Si l'on observe le champ lexical du miroloï, la différenciation est évidente. Les verbes employés pour qualifier l'action de la pleureuse sont principalement: pleurer (kléo), crier (fonazo), dire (léo) ou mirologiser (mirologo). En aucun cas, l'on ne «chante» un mirolö̈.

Les lamentations que j'ai pu enregistrer, soit lors d'un rituel de commémoration, soit à ma demande dans l'intimité d'un salon ou d'une cuisine, reposaient pour la plupart sur la même mélodie se déployant sur un petit nombre de notes contenues dans un ambitus restreint. Ainsi, sur un même schéma mélodico-rythmique, les pleureuses improvisent des vers à quinze pieds (décapentosyllabiques). Quant aux textes, leur contenu est improvisé à partir d'un stock de formules types. On retrouve à travers toute la Grèce les mêmes schémas : la pleureuse chante les louanges du défunt, mais aussi l'accuse d'avoir abandonné sa famille. Elle s'adresse aussi à l'assemblée réunie autour du défunt, parfois parle au nom des parents du défunt. Par sa voix, le mort interpelle les vivants (Xanthakou, 1990) et répond aux questions qui lui sont posées ${ }^{3}$.

Sans entrer dans les détails de l'analyse de l'articulation du rapport musique-texte, la caractéristique stylistique principale qui se dégage est que les femmes insèrent à l'intérieur même des mots des syllabes sans signification. Celles-ci, placées toujours à la même place mélodique, rendant par endroits difficile, voire impossible la compréhension du texte, introduisent du désordre du point de vue du sens. Mais du point de vue mélodique, ces syllabes agissent comme une sorte de refrain. Ces sanglots stylisés chantés en voix de fausset interviennent avec régularité tout en portant une grande intensité dramatique.
S’il est possible de décrire les lamentations en des termes musicaux, ces dernières ne sont pas pensées comme faisant partie de la catégorie musique, au même titre que le reste de l'ensemble du répertoire des femmes. Elles occupent une place bien particulière tant du point de vue musical que social. Elles sont nécessaires lors des rituels funéraires mais, hors rituel, leur pratique est condamnée par les hommes car elles sont jugées comme potentiellement dangereuses. un violoniste, un luthiste, un accordéoniste et un joueur de défi (tambour sur cadre). Ce sont des ensembles familiaux de tradition orale organisés autour d'un chef, le clarinettiste. Cette hiérarchie organisationnelle se retrouve dans la musique elle-même. Le clarinettiste est le soliste principal et dirige l'ensemble des musiciens qui lui offrent principalement un soutien harmonique.

Le paniyiri dure entre une, deux ou trois nuits successives suivant l'importance du

LA CARACTÉRISTIQUE STYLISTIQUE PRINCIPALE QUI SE DÉGAGE EST QUE LES FEMMES INSÈRENT À L'INTÉRIEUR MÊME DES MOTS DES SYLLABES SANS SIGNIFICATION. CELLES-CI, PLACÉES TOUJOURS À LA MÊME PLACE MÉLODIQUE, RENDANT PAR ENDROITS DIFFICILE, VOIRE IMPOSSIBLE LA COMPRÉHENSION DU TEXTE, INTRODUISENT DU DÉSORDRE DU POINT DE VUE DU SENS.

\section{LE MIROLOÏ INSTRUMENTAL, LA LAMENTATION DANS LES FÊTES}

On l'a dit en introduction, le terme mirolö̈ est aussi employé pour désigner des lamentations instrumentales jouées dans un contexte très différent, à savoir lors des paniyiria, fêtes patronales.

Ces miroloyia n'ont pas le caractère de dangerosité des lamentations des femmes. Les évoquer ne provoque pas d'effroi mais bien plutôt des réactions de respect presque solennelles : «to miroloï iné proto, édho iné tin psichi mas» (Le miroloï est premier, c'est là qu'est notre âme), comme me le confia un Épirote d'une cinquantaine d'années.

Avant de s'interroger sur la place et la signification du mirolö̈ dans la fête patronale, il est nécessaire de donner une brève description de celle-ci.

Le paniyiri constitue l'un des événements majeurs de la vie des villages de l'Épire. La région étant une terre d'exil massif ${ }^{4}$, ces villages sont presque vides pendant la plus grande partie de l'année. En revanche, durant l'été, la vie locale s'intensifie brusquement avec le retour des émigrés et connaît son apogée les jours de paniyiri. Un village de quarante habitants à l'année peut accueillir jusqu'à deux à trois cents personnes à sa fête. Ainsi, l'immense majorité des participants aux paniyiria ne sont pas des villageois mais des citadins, pour beaucoup vivant à l'étranger ${ }^{5}$.

Les fêtes sont animées par des koumpanies, ensembles de musiciens tsiganes qui comprennent un, voire deux clarinettistes, village. Après une messe matinale en l'honneur du saint patron, les villageois se rassemblent sur la place du village pour dîner puis danser et chanter tout au long de la nuit. Le principe général de la fête est que se succèdent les parées, groupes familiaux ou d'amis, au centre de la place pour danser en formant un cercle ouvert emmené par un premier danseur. C'est ce protochoreftis, souvent chef de famille, qui commande aux musiciens ce qu'il veut danser contre de l'argent. Les musiciens se trouvent donc rémunérés progressivement, morceau après morceau ${ }^{6}$. D'une façon schématique, l'on peut dire que les fêtes qui fonctionnent bien ${ }^{7}$ obéissent à un même déroulement type. L'on passe d'une atmosphère assez protocolaire pendant laquelle dansent les organisateurs de la fête et les notables, puis progressivement les danseurs se succèdent de façon moins formelle et les danses se font plus rapides. Enfin, la fatigue et l'alcool aidant, l'on revient à des pulsations plus lentes ainsi qu'à des épitrapezia, chants de table et des lamentations 8 .

Musicalement, le mirolö̈ instrumental semble très différent des lamentations vocales. À l'économie de notes de ces dernières s'oppose une profusion. Le clarinettiste déploie une mélodie construite autour de formules types développées au-dessus d'un bourdon tenu par le reste des instrumentistes. Puis les rôles s'échangent, le second clarinettiste prend à son tour la voix soliste avant de la passer au violoniste qui lui-même la redonne au premier clarinettiste. La pièce, sans pulsation régulière, 
frappe par le très grand contraste qu'elle offre entre des notes tenues, exposées avec lenteur et qui dévoilent une structure pentatonique anhémitonique et une riche ornementation dense et rapide qui s'écarte très largement du mode. Par son caractère non mesuré, le miroloï se distingue du reste du répertoire joué aux fêtes.

\section{LE MIROLOÏ ENCADRE LA FÊTE}

À l'intérieur de ce déroulement type, le mirolö̈ occupe une place centrale dans la fête. C'est par une série de lamentations que celle-ci s'ouvre et se termine.

Alors que la majorité des villageois est déjà installée et que le repas a débuté, les musiciens ouvrent la fête par une série de miroloyia instrumentaux. Par la suite, ce sont les villageois qui commandent les pièces qu'ils veulent entendre ou danser, ici personne n'a donné l'ordre de jouer une lamentation. Cela va de soi, tout paniyiri doit débuter ainsi.

Soufflant à pleins poumons, leurs instruments presque à l'horizontale, les clarinettistes semblent vouloir remplir tout l'espace sonore. Ils s'adressent à l'ensemble des villageois. Ces derniers, attablés, mangent, boivent et discutent. D'autres se déplacent pour commander leur repas, certains s'interpellent d'une table à l'autre. Il règne une atmosphère de convivialité détendue. Pour autant, si l'on s'attarde davantage sur les visages, l'on s'aperçoit que de façon fugace mais répétée, le regard de quelqu'un d'enjoué se brouille un instant; ou encore qu'un sourire devient subitement grave avant que de se terminer en éclat de rire. De temps à autre, certains regardent en direction des musiciens mais sans insistance. Ces comportements ne signifient pas que les villageois ne prêtent pas attention à la musique mais bien plutôt que celle-ci n'est pas dissociée du reste de la fête. Les musiciens poursuivent les lamentations par quelques danses dont les textes évoquent l'exil. Puis les premiers danseurs arrivent sur la place et les danses peuvent commencer.

Sauf problème, on ne revient pas aux lamentations avant la dernière partie de la fête. Cela peut avoir lieu lorsque, pour une raison ou pour une autre, personne n'est venu demander de danses aux musiciens depuis au moins une demi-heure. Ceux-ci, de leur propre initiative, jouent un mirolo $i$ qu'ils font suivre d'une suite de danses du même type qu'au début de la fête. Il s'agit là pour eux de tenter de relancer la fête en l'« ouvrant» une seconde fois.

En revanche, de trois ou quatre heures du matin jusqu'à l'aube, les miroloyia sont attendus. L'atmosphère change progressivement au fur et à mesure que la place se vide. Il ne reste bientôt plus qu'un petit groupe d'irréductibles, une trentaine de personnes, qui décidément ne veulent pas que la fête se termine. Petit à petit, ils commandent davantage de chants de table et de miroloyia que de danses.

Ce sont les hommes qui demandent des lamentations. Les femmes sont, elles, plus en retrait. Contrairement au début de la fête, la lamentation est personnalisée : elle a pour destinataire premier celui qui l'a commandée et payée aux musiciens. Un bon musicien doit connaître les goûts de chaque villageois et improviser en fonction de cela.

\section{C'EST PARADOXALEMENT}

\section{DANS UN CONTEXTE DE FÊTE}

\section{QUE LES HOMMES}

\section{PLEURENT LEURS DÉFUNTS.}

C'est un moment de la fête où les hommes se laissent aller à leurs émotions et pleurent. Ainsi, par exemple, un homme lance aux musiciens, la voix enivrée: «Un miroloï! Un mirolö̈! et avec cœur, avec cœur», avant que de s'affaler sur sa chaise sans quitter le clarinettiste des yeux. Celui-ci se lève, vient se poster face à lui et entame la lamentation avec énergie. L'homme lui crie: «Pio siga! siga!» (plus lentement! lentement). Immédiatement, le musicien s'exécute et l'homme lui jette un billet de cinquante euros. Le clarinettiste approche le pavillon de sa clarinette au plus près de l'oreille du commanditaire, lequel sourit et pleure en même temps. Ses amis l'embrassent comme pour le soutenir. D'autres, dans l'assemblée, ont les yeux embués ou le visage grave. Musicalement, l'interprétation du mirolö̈ a changé par rapport à celle qu'en faisaient les musiciens en ouverture. Plus de grand volume sonore destiné à remplir toute la place mais un son beaucoup plus doux et intime. À l'écoute d'enregistrements, sans aucune indication, il est tout à fait possible de savoir s'il s'agit d'une lamentation de début ou de fin de fête tant l'esthétique musicale est différente.

Plus le paniyiri avance et plus les villageois alternent entre chants de table et lamentations. Enfin, l'aube arrivant, un homme s'adresse aux musiciens: «Eh! Un mirolö̈! Que l'on ferme! [ ] Un mirolö̈ et l'on va se coucher! », il insiste: "Allez! Un dernier! que l'on ferme! », et ajoute: «On va pleurer et après on veut rien d'autre!» Il s'agit là du dernier morceau exécuté. À la différence des lamentations précédentes, ce mirolö̈, s'il est commandé par un homme, s'adresse à l'ensemble des vil- lageois réunis. Cela est très clair, si l'on observe les attitudes d'écoute de chacun et le comportement des musiciens.

Ainsi, la fête se termine-t-elle comme elle avait commencé. Un patron de taverne avec qui je m'entretenais de la fête de la veille employa cette image: "ça fait un cercle» (kani éna kiklo).

\section{L'ÉVOCATION DES MORTS ET DES EXILÉS}

Un excellent clarinettiste à qui je demandais pourquoi les paniyiria débutaient toujours par des lamentations me répondit:

C'est pour que les gens se rassem-

blent. D'abord le mirolö̈, quoi qu'il arrive Après seulement différentes chansons Ça doit être en premier le miroloï, et après les gens se rassemblent, un miroloï, un deuxième et un troisième jusqu'à ce que tout le monde soit là et la fête commence.

- Le miroloï vous le jouez pour les morts?

- Non, non, non, pour les exilés ou les absents, ceux qui partent loin, qui partent pour le travail.

- Pas pour les morts? Moi, je croyais que c'était pour les morts et les exilés.

Il me coupe, irrité :

- Non, il y a des miroloyia pour les morts mais aussi des miroloyia pour ceux qui partent en exil et qui reviennent ici, tu comprends.

- Et les miroloyia qui sont pour les morts, ce sont lesquels alors?

- I mariola.

- Ah oui et vous le jouez celui-là?

- Nous le jouons

finit-il par consentir, tout en s'éloignant.

Il s'agit justement d'un miroloï qui est joué au début des fêtes. Pendant le temps qu'a duré notre échange, Yorgos était plutôt nerveux. Il semblait avoir hâte que je cesse de le questionner à propos des morts. Des exilés, il voulait bien parler, des morts, non.

Cependant, alors que je questionnais une pleureuse à ce sujet, elle me dit: «C'est pour ceux qui manquent»; tout en désignant d'un coup de menton le mur de son salon. Elle venait de désigner la direction du cimetière. Ainsi, le mirolö̈ est-il un répertoire destiné à tous les absents, que leur absence soit définitive, les morts, ou temporaire, les exilés ${ }^{9}$. Une fête ne peut commencer autrement que par leur évocation.

La plupart du temps, les gens ne disent pas à qui ils pensent durant la lamentation; les choses sont tacites : chacun connaît les morts et les exilés des autres. Cependant, 
cela est parfois précisé: comme cet homme qui dit à un clarinettiste: «Joue-le pour mon neveu », avant de se mettre à pleurer.

L'observation des interactions entre les musiciens et le commanditaire nous renseigne sur l'esthétique du mirolö̈. Les ordres qui sont passés parfois sur un ton plus qu'impératif vont toujours dans le sens d'une interprétation plus lente, siga, plus douce, gliko, mais aussi plaintive (paraponéméno), triste (lipitéro) et enfin vari, que l'on peut traduire par lourd ou pesant.

Exceptés les deux premiers, ce sont des termes qui sont aussi employés pour qualifier les miroloyia des femmes. De même que les verbes kléo (pleurer) et léo (dire) s'appliquent aussi bien à l'action de la pleureuse qu'à celle du clarinettiste. Pour autant, les sentiments à l'écoute des miroloyia instrumentaux ont quelque chose de plus léger: «Il y a de la légèreté dedans. Ce n'est pas que lourd» (Exhoun alafria mésa Dhen iné olo vari) m'explique un homme d'une quarantaine d'années. Ce que confirme un musicien ainsi: "C'est de la tristesse et de la joie Ce sont des larmes de joie» (Iné lipi ké xharas Iné klama tis xharas).

Le terme mirolö̈ désigne en définitive des réalités fort différentes. On y entrevoit une différenciation des rôles des deux sexes face à la mort. D'un côté, les miroloyia vocaux sont le fait de femmes qui pleurent les défunts lorsque la mort vient de surgir. Ce sont elles qui gèrent l'irruption de la mort et qui font en sorte que l'âme prenne le bon chemin. Les hommes, eux, sont alors bien en retrait, voire absents. Au quotidien, ils réprouvent la pratique hors rituel de ces lamentations dans la mesure où elles introduisent le désordre et risquent d'attirer Kharos dans la maison.

C'est paradoxalement dans un contexte de fête que les hommes pleurent leurs défunts. On est là dans un temps distancié par rapport à l'évènement de la mort. Contrairement aux femmes, les hommes ne s'adressent pas directement à leurs morts. Ils passent par un intermédiaire: le musicien tsigane. Celui-ci, dont le statut en marge de la société n'est certainement pas innocent dans cette problématique, a la charge de gérer leurs émotions. Le clarinettiste doit les faire pleurer sans pour autant leur faire perdre le contrôle d'eux-mêmes. Par sa musique, construite en interaction avec le destinataire premier, sont évoqués les défunts et les absents.

Le paniyiri, seul moment de l'année où la communauté villageoise est réunie, est le théâtre de réajustements des liens sociaux et des rapports de forces. Chaque famille réaffirme sa présence dans le village malgré l'exil. L'évocation des ancêtres par la lamentation y est alors décisive. Il semble bien qu'en Épire, faire la fête, c'est d'abord se souvenir de ses défunts.

\section{Bibliographie}

ALEXIOU, M. (1974). The Ritual Lament in Greek Tradition, Cambridge, Cambridge University Press.

AUERBACH, S. (1987). «From singing to lamenting: Women's musical role in a Greek village », dans Ellen KOSKOFF (dir.), Women and Music in a Cross Cultural Perspective, New York, Greenwood Press, p. 25-45.

CARAVELLI-CHAVES, A. (1986). «The bitter wounding: The lament as a social protest in rural Greece », dans Gender and Power in Rural Greece, Princeton (N. J.), Princeton University Press, p. 169-195.

CARAVELLI-CHAVES, A. (1980). «Bridge between worlds : the Greek women's lament as a communicative event ", Journal of American Folklore, no 93, p. 129-157.

DAMIANAKOS, S. (1996). Le paysan grec: défis et adaptations face à la société moderne, Paris, L'Harmattan.

DANFORTH, L. (1982). The Death Rituals of Rural Greece, photographies de Alexander TSIARAS, Princeton (N.J.), Princeton University Press.

DELAPORTE, H. (2007 [sous presse]). "Quand les chanteurs sont grecs et les musiciens tsiganes. La musique traditionnelle en Épire », Études balkaniques.

HERZFELD, M. (1981). "Performative categories and symbols of passage in rural Greece", Journal of American Folklore, no 94 , p. 44-57.

KAKABURA-TILI, R. (1999). Avó $\mu \varepsilon \sigma \alpha \sigma \tau o$

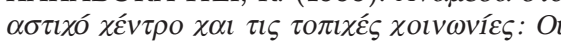

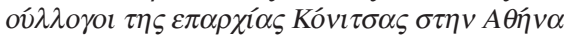
(Entre centre urbain et sociétés locales: les associations régionales de Konitsa siégeant à Athènes), Konitsa, Édition du Centre culturel municipal de Konitsa.

MOTSISOS, Y. (2000). To eliniko miroloyi (Le miroloï grec), Athènes, Kodhikas.

SAUNIER, G. (1999). Elinika dhimotica traghoudhia: ta miroloyia (La chanson populaire grecque: les miroloyia), Athènes, Nepheli.

SEREMENTAKIS, N. (1991). The Last Word. Women, Death and Divination in Inner Mani, Chigago, University of Chicago Press.

XANTHAKOU, M. (1990). "Discours d'outretombe: le langage du mirolö̈», Cahiers de littérature orale, $\mathrm{n}^{\circ}$ 27, Paris, INALCO, p. 139-162.

\section{Notes}

1. Cet article est basé sur des données de terrain recueillies à diverses occasions depuis 1999 dans des villages du Nord de l'Épire, près de la frontière albanaise.

2. Ces données viennent de sources bibliographiques et de propos de villageois que nous avons interrogés à ce sujet. Nous n'avons pas assisté nous-même à un tel rituel.

3. Pour davantage d'informations concernant les situations d'élocution et les revendications dans les lamentations, se reporter à Caravelli, 1986.

4 D'une émigration saisonnière sous l'Empire ottoman, l'on est passé à une émigration définitive dès la fin du XIX ${ }^{\mathrm{e}}$ siècle. Par vagues successives, les villageois sont partis chercher du travail dans les grandes villes de Grèce (Athènes, Thessalonique, Igoumenitsa, Ioannina, Agrinion), mais aussi à l'étranger, principalement en Allemagne, aux États-Unis et en Australie (Damianakos, 1996, p. 87-121).

5. Ils sont très fortement attachés à leur village d'origine et tiennent à participer, même à distance, à sa vie sociale, notamment par le biais d'associations (Kakabura-Tili, 1999).

6. Les prix sont libres. Il n'est pas rare de payer pour une suite de danses d'une vingtaine de minutes jusqu'à deux euros. Un chef de famille peut dépenser jusqu'à mille euros.

7. Qu'un décès survienne dans une famille peu avant le paniyiri et c'est toute une partie du village, du fait des liens de parenté, qui ne peut pas danser.

8. Pour davantage de précisions sur le déroulement des paniyiria et leurs enjeux, voir Delaporte, 2007.

9. Une partie de notre thèse en cours de rédaction est consacrée à la façon dont les Épirotes articulent mort et exil. Sur ce thème, consulter l'article d'Estelle Amy de la Bretèque (p. 60) dans ce même numéro. 